\title{
Network pharmacology approach to evaluate the therapeutic effects of mulberry leaf components for obesity
}

\author{
GUIDAN WANG and JINE DONG \\ Health Management Research Laboratory, Hunan Future Health Technology Group Co., Ltd. \\ (Future Health University), Changsha, Hunan 410000, P.R. China
}

Received March 9, 2021; Accepted June 28, 2021

DOI: $10.3892 /$ etm.2021.10978

\begin{abstract}
Obesity is a chronic condition that has become a serious public health challenge globally due to the association with a high incidence of complications. Mulberry leaf is one of the most commonly used medicinal and herbal medicines that has been reported to ameliorate obesity and hyperlipidemia. However, the mechanism remains unclear. In the present study, a network pharmacology approach was used to explore the potential mechanism underlying the effects of mulberry leaf extract on obesity. First, the potential targets of mulberry leaf and obesity were predicted using SwissTargetPrediction, Online Mendelian Inheritance in Man, GeneCards and Comparative Toxicogenomics Database databases, which were then used to construct the protein-protein interaction networks. Gene ontology (GO) enrichment and Kyoto Encyclopedia of Genes and Genomes (KEGG) enrichment analyzes were performed using $\mathrm{R}$ version 3.6.3. Finally, results of this network analysis were verified by using the mulberry leaf extract to treat high-fat diet-induced obese mice. In total, 24 target genes associated with obesity that could potentially be affected by mulberry leaf treatment were predicted by network pharmacology, using which top seven related pathways were determined by KEGG enrichment analysis. Triglyceride (TG) and total cholesterol (TC) levels in mice serum were detected using TG and TC assay kits. Hepatic fat accumulation was detected by H\&E staining whereas liver lipid droplets were detected by Oil red $\mathrm{O}$ staining in mice tissues. The expression of IL-1 $\beta, N F-\kappa B$ inhibitor $\alpha$, inducible nitric oxide synthase, AMP-activated protein kinase (AMPK), sterol regulatory element-binding proteins and fatty acid synthase in the visceral white adipose tissues of mice was analyzed by western blotting. The expression of TNF- $\alpha$, peroxisome proliferator activated
\end{abstract}

Correspondence to: Dr Jine Dong, Health Management Research Laboratory, Hunan Future Health Technology Group Co., Ltd. (Future Health University), 792 Section 4 Furong South Road, Muyun Street, Changsha, Hunan 410000, P.R. China

E-mail: dongjine2005@163.com

Key words: mulberry leaf components, obesity, network pharmacology, target, protein interaction receptor (PPAR)D, PPARG, fatty acid amide hydrolase (FAAH) and hydroxysteroid 11- $\beta$ dehydrogenase 1 (HSD11B1) in the visceral white adipose tissues of mice was detected by reverse transcription-quantitative PCR. Mulberry leaf extract was found to reduce fat accumulation and hepatic lipid droplet formation. Mulberry leaf also alleviated inflammation and lipogenesis whilst promoting lipid catabolism and fatty acid oxidation by promoting the AMPK signaling pathway. The possible anti-obesity effects of mulberry leaf on the mice may be due to the downregulation of TNF- $\alpha$, PPARD and PPARG and the upregulation of FAAH and HSD11B1. These results were consistent with the GO enrichment analysis and suggested that mulberry leaf may regulate lipid metabolism and catabolism, fatty acid metabolism and biosynthesis and the inflammatory response to reduce obesity.

\section{Introduction}

Obesity is a chronic metabolic condition closely associated with lifestyle (1). According to previous epidemiological survey data, male and female individuals with a BMI $>28 \mathrm{~kg} / \mathrm{m}^{2}$ in China account for 17.10 and $13.37 \%$ of the total population as of 2017, respectively, where a clear upward trend can be observed (2). Obesity is a risk factor for type 2 diabetes (3), cardiovascular and cerebrovascular diseases (4) and a variety of cancers (5). To improve human health and the quality of life, it is necessary to fundamentally determine the pathogenesis of obesity to design curative treatments.

Mulberry leaf extract (MLE) is a well-known herb that is widely used as a medicine or for food consumption in China (6). It has been previously reported that MLE and its extracts can exert a variety of therapeutic effects, including the pharmacological effects of lowering blood glucose (7) and blood lipid levels (8), anti-diabetic (9), antioxidant (10) and antitumor properties (11). In a previous study, Zeni and Dall'Molin (12) treated a total of 33 hyperlipidemic Wistar rats with a water extract of MLE following the oral administration of a cholesterol-rich ( $1 \mathrm{~g} / 100 \mathrm{~g}$ body weight) diet. After 14 days, the concentrations of triglyceride (TG), total cholesterol (TC) and low-density lipoprotein in the MLE treatment group were reduced compared with those in the hyperlipidemic control group. In another study, Lee et al (13) revealed that the water extract of MLE could ameliorate atherosclerosis and hypertension, hyperlipidemia and vascular dysfunction in rats with atherosclerosis. 
In addition, MLE extracts have been reported to alleviate non-alcoholic fatty liver disease in high-fat diet (HFD)-fed mice by suppressing adipocytokines, inflammation and oxidative stress (14). MLE also markedly reduced obesity in mice induced by feeding on a high-fat diet by increasing the activity of brown adipose tissues (15). Li et al (16) previously demonstrated that MLE polyphenols and fibers confer synergistic effects on weight loss and the regulation of the intestinal flora and metabolites. However, to the best of our knowledge, the mechanism by which MLE inhibits adipogenesis remains poorly understood.

In the present study, genetic or protein targets of active components contained within MLE towards obesity were identified using bioinformatics analysis. These target components by MLE for the treatment of obesity were verified using network pharmacology, including target prediction, protein-protein interaction (PPI), ingredients-targets network, Gene Ontology (GO) terms and Kyoto Encyclopedia of Genes and Genomes (KEGG) pathway enrichment analyzes. Finally, in vivo experiments using a mouse model of obesity induced by a high-fat diet were performed to assess the effect of the key compounds derived from network pharmacology on obesity.

\section{Materials and methods}

Experimental design. A detailed experimental design of the present study is shown (Fig. 1).

Collection of chemical ingredients of MLE. To determine the chemical ingredients in MLE, the Traditional Chinese Medicine Systems Pharmacology (TCMSP; version 2.3; https://old. tcmsp-e.com/tcmsp.php) was performed by searching with the key words 'Sangye' or 'Mori Follum'. The screening conditions were as follows: Oral bioavailability $(\mathrm{OB}) \geq 30 \%$; Drug-likeness $(\mathrm{DL}) \geq 0.18$ and Caco-2 permeability $($ Caco- 2$)>0$.

Targets of MLE and obesity. The herb targets were predicted by the SwissTargetPrediction website (https://www.swisstargetprediction.ch/) through the structures of active ingredients in MLE selected from TCMSP (Fig. S1). The disease targets were searched using 'obesity' as the key word through Online Mendelian Inheritance in Man (OMIM; https://www.omim. org/), GeneCards (version 5.3; https://www.genecards.org/) and Comparative Toxicogenomics Database (CTD; https://ctdbase. org/).

Potential targets of MLE in the treatment of obesity. The herb targets were intersected with the disease targets to obtain the candidate targets of MLE in obesity by Venny (version 2.1; https://bioinfogp.cnb.csic.es/tools/venny/index.html), which were defined as MLE-obesity related targets. These targets were uploaded into the Search Tool for the Retrieval of Interacting Genes/Proteins (STRING) database (version 11.0; https://string-db.org/) to establish a PPI network. The ingredients-targets network was constructed by linking the active compounds with their potential targets using Cytoscape (version 3.7.2; https://cytoscape.org/).

GO gene enrichment analysis and KEGG pathway annotation. To examine the biofunctions of the 234 genes, the 'clusterProfiler' package (17) in R software (version 3.6.3) was used to perform functional annotation, which included the three categories of GO (biological processes, molecular functions and cellular components) and KEGG enrichment analysis (https://www.r-project.org/). The parameters were set at $\mathrm{P}=0.05$ cutoff and $\mathrm{q}=0.05$ cutoff. The results were visually presented using the 'GOplot' package (18).

High-fat diet-induced obesity mouse model. In total, 20 male C57BL/6 mice (4 weeks old; $20.01 \pm 0.24 \mathrm{~g}$ ) were provided by Hunan SJA Laboratory Animal Co., Ltd (Hunan, China). Mice were housed under a constant temperature $\left(22 \pm 2^{\circ} \mathrm{C}\right), 50-70 \%$ relative humidity and 12-h light/dark cycle with ad libitum access to food and clean water for 1 week. After 1 week of adaptation, 20 mice were randomly divided into the following four groups $(\mathrm{n}=5)$ : i) Normal control diet group (Control); ii) obese high-fat diet group (Model); iii) obese mice receiving a low dose (133 mg/kg) of MLE group; and iv) obese mice receiving a high dose $(666 \mathrm{mg} / \mathrm{kg} /$ day $)$ of MLE group. The doses for low-dose and high-dose of MLE was determined according to a previous study (19).

MLE was freshly suspended in distilled water and mice were administered via oral gavage five times a week not during the weekend for 8 weeks. The Control and Model groups were given the same volumes of distilled water. At the end of experiment at week 8 , mice were weighed and sacrificed using an intraperitoneal injection of $150 \mathrm{mg} / \mathrm{kg}$ pentobarbital sodium, before their blood, liver tissues and visceral white adipose tissues were collected immediately and stored at $-80^{\circ} \mathrm{C}$. The experimental protocols were approved by the Institutional Animal Care and Use Committee of the Hunan Future Health Technology Group Co., Ltd. (Changsha, China).

Serum analysis. Blood was centrifuged at $500 \mathrm{x} \mathrm{g}$ for $15 \mathrm{~min}$ at $4^{\circ} \mathrm{C}$ to collect the serum. Commercial analysis kits (Abcam) were used to detect the levels of serum TG (cat. no. ab65336) and TC (cat. no. ab282928).

$H \& E$ staining. The liver tissues frozen in liquid nitrogen were taken out, fixed in $10 \%$ neutral buffered formalin at room temperature for $24 \mathrm{~h}$ before being embedded in paraffin wax. The liver tissue sections $(4 \mu \mathrm{m})$ were then cut and hydrated in a decreasing ethanol gradient. All sections were deparaffinized with xylene and stained with hematoxylin for $5 \mathrm{~min}$ at room temperature and eosin for $3 \mathrm{~min}$ at room temperature. The sections were finally observed under a light microscope (magnification, x200; OCT-HS100; Canon, Inc.).

Oil red $O$ staining. The liver tissue sections $(4 \mu \mathrm{m})$ were stained with Oil red $\mathrm{O}$ according to the manufacturer's protocol of the Oil Red O Staining Kit (cat. no. C0157S; Beyotime Institute of Biotechnology). Oil red O-stained sections were imaged using an inverted light microscope (magnification, x400; Nikon Corporation).

Western blot analysis. Total proteins were extracted from the visceral white adipose tissues using RIPA reagent (Beyotime institute of Biotechnology) on ice. The protein concentration was determined using a BCA kit. Equal amounts $(20 \mu \mathrm{g})$ of protein were loaded and separated by $10 \%$ SDS-PAGE. After electrophoresis, proteins were transferred onto PVDF 


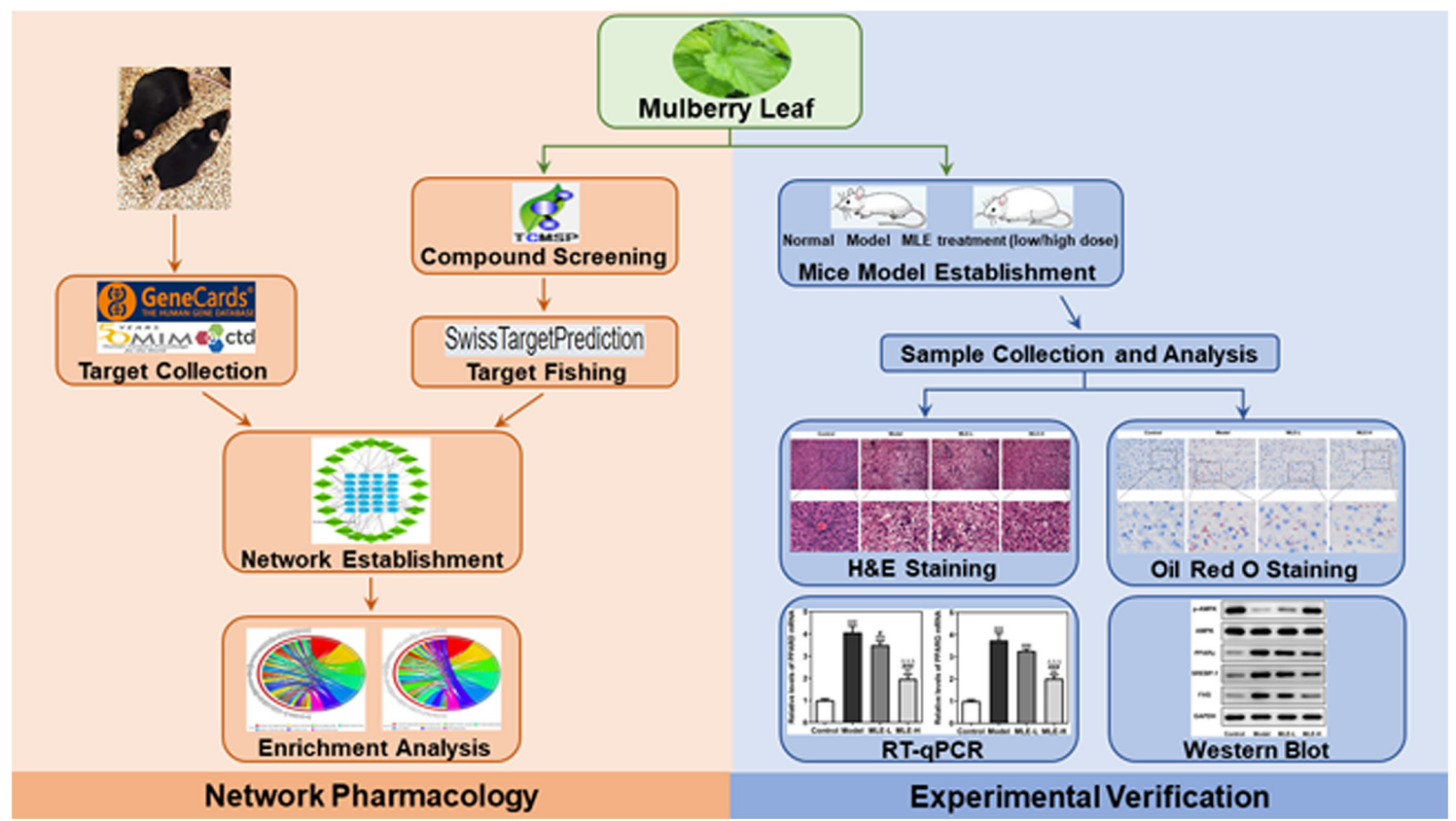

Figure 1. Flowchart illustrating the network analysis approach and study design. RT-qPCR, reverse transcription-quantitative PCR.

membranes and the membranes were blocked with $5 \%$ non-fat milk for $2 \mathrm{~h}$ at $37^{\circ} \mathrm{C}$. Subsequently, the membranes were immunoblotted with the following primary antibodies overnight at $4^{\circ} \mathrm{C}$ : TNF- $\alpha$ (dilution, 1:1,000; cat. no. ab183218; Abcam), IL-1 $\beta$ (dilution, 1:1,000; cat. no. ab234437; Abcam), NF-кB inhibitor $\alpha$ (NFKBIA; dilution, 1:1,000; cat. no. ab32518; Abcam), inducible nitric oxide synthase (iNOS; dilution, 1:1,000; cat. no. ab178945; Abcam), AMP-activated protein kinase (AMPK; dilution, 1:2,000; cat. no. 5832; Cell Signaling Technology, Inc.), phosphorylated (p-) AMPK (dilution, 1:2,000; cat. no. 5759; Cell Signaling Technology, Inc.), peroxisome proliferator activated receptor (PPAR)- $\gamma$ (PPARG; dilution, 1:500; cat. no. ab45036; Abcam), sterol regulatory element-binding proteins (SREBP-1; dilution, 1:1,000; cat. no. ab28481; Abcam), fatty acid synthase (FAS; dilution, 1:1,000; cat. no. ab82419; Abcam) and GAPDH (dilution, 1:2,500; cat. no. ab9485; Abcam). This was followed by incubation with the HRP-conjugated secondary antibody (cat. no. 7074; dilution, 1:1,000; Cell Signaling Technology, Inc.) at room temperature for $1 \mathrm{~h}$. The protein bands were visualized using enhanced chemiluminescence reagent (Research-bio). Image Lab 3.0 software (Bio-rad laboratories, inc.) was used to quantify the WB densitometry.

Reverse transcription-quantitative PCR analysis. Total RNA from the visceral white adipose tissues was isolated using the TRIzol ${ }^{\circledR}$ reagent (Thermo Fisher Scientific, Inc.). Subsequently, total RNA was converted into cDNA using PrimeScript 1st strand cDNA Synthesis Kit (cat. no. 6110A; Takara Bio, Inc.). The reverse transcription condition was as follows: $10 \mathrm{~min}$ at $30^{\circ} \mathrm{C}$ and $40 \mathrm{~min}$ at $42^{\circ} \mathrm{C}$. Quantitative PCR was performed using SYBR ${ }^{\circledR}$ Premix Ex Taq ${ }^{\mathrm{TM}}$ (Takara Bio, Inc.) with an ABI Prism 7900 machine (Applied Biosystems; Thermo Fisher Scientific, Inc.). The internal control was GAPDH. Amplification was performed as follows: $5 \mathrm{sec}$ at $95^{\circ} \mathrm{C}$, followed by 40 cycles of $15 \mathrm{sec}$ at $95^{\circ} \mathrm{C}$ and $40 \mathrm{sec}$ at $56^{\circ} \mathrm{C}$. The relative gene expression levels of TNF $\alpha$, PPARD, PPARG, fatty acid amide hydrolase (FAAH) and hydroxysteroid 11- $\beta$ dehydrogenase 1 (HSD11B1) were calculated using the $2^{-\Delta \Delta \mathrm{Cq}}$ method (20). Primers sequences were as follows: TNF $\alpha$ forward, 5'-AGCCCATGTTGTAGCAAACC-3' and reverse, 5'-GGAAGACCCCTCCCAGATAG-3'; PPARD forward, 5'-ACGCACCCTTTGTCATCC-3' and reverse, 5'-GAAGAGGCTGCTGAAGTTGG-3'; PPARG forward, 5'-GAGAAGGAGAAGCTGTTGGC-3' and reverse, 5'-ATG GCCACCTCTTTGCTCT-3'; FAAH forward, 5'-GCCTCA AGGAATGCTTCAGC-3' and reverse, 5'- TGCCCTCATTCA GGCTCAAG-3'; HSD11B1 forward, 5'-AAGCAGACCAAC GGGAGCATT-3' and reverse, 5'- GGAGAAGAACCCATC CAGAGCA-3' and GAPDH forward, 5'-TCTTGCTCAGTG TCCTTGC-3' and reverse, 5'-CTTTGTCAAGCTCATTTC CTGG-3'.

Statistical analysis. Data are presented as the mean \pm SD with five mice per group. The significance of differences was determined by one-way ANOVA followed by Tukey's post hoc test for multiple comparisons using SPSS v22.0 (IBM Corp.). $\mathrm{P}<0.05$ was considered to indicate a statistically significant difference.

\section{Results}

Active components and potential target proteins of MLE. TCMSP was used to retrieve the composition of MLE and there were 26 active components when the filter conditions were as follows: $\mathrm{OB} \geq 30 \%, \mathrm{DL} \geq 0.18$ and $\mathrm{Caco} 2>0$ (Table I and Fig. S1). Using SwissTargetPrediction, 521 herb targets were predicted. From the analysis using OMIM, GeneCards and CTD, 190 obesity-related genes were retrieved from OMIM, 8,757 obesity-related genes were retrieved from GeneCards and 31,922 obesity-related genes were retrieved from CTD. The disease targets were identified by the intersection of genes retrieved from the three databases, where 
Table I. Mol ID of the 26 active components found in mulberry leaves.

\begin{tabular}{|c|c|c|c|c|}
\hline Mol ID & Molecule name & Oral bioavailability (\%) & Drug likeness & Caco-2 permeability \\
\hline MOL001771 & Poriferast-5-en-3 $\beta$-ol & 36.91 & 0.75 & 1.45 \\
\hline MOL002773 & $\beta$-carotene & 37.18 & 0.58 & 2.25 \\
\hline MOL003842 & Albanol & 83.16 & 0.24 & 0.41 \\
\hline MOL003847 & Inophyllum E & 38.81 & 0.85 & 0.68 \\
\hline MOL003850 & 26-Hydroxy-dammara-20,24-dien-3-one & 44.41 & 0.79 & 0.82 \\
\hline MOL003851 & Isoramanone & 39.97 & 0.51 & 0.05 \\
\hline MOL003856 & Moracin B & 55.85 & 0.23 & 0.83 \\
\hline MOL003857 & Moracin C & 82.13 & 0.29 & 0.87 \\
\hline MOL003858 & Moracin D & 60.93 & 0.38 & 1.03 \\
\hline MOL003859 & Moracin E & 56.08 & 0.38 & 0.96 \\
\hline MOL003860 & Moracin F & 53.81 & 0.23 & 0.81 \\
\hline MOL003861 & Moracin G & 75.78 & 0.42 & 0.98 \\
\hline MOL003862 & Moracin $\mathrm{H}$ & 74.35 & 0.51 & 0.87 \\
\hline MOL003879 & 4-Prenylresveratrol & 40.54 & 0.21 & 0.9 \\
\hline MOL000729 & Oxysanguinarine & 46.97 & 0.87 & 1.08 \\
\hline MOL000098 & Quercetin & 46.43 & 0.28 & 0.05 \\
\hline MOL000358 & $\beta$-sitosterol & 36.91 & 0.75 & 1.32 \\
\hline MOL000422 & Kaempferol & 41.88 & 0.24 & 0.26 \\
\hline MOL000449 & Stigmasterol & 43.83 & 0.76 & 1.44 \\
\hline MOL001439 & Arachidonic acid & 45.57 & 0.2 & 1.2 \\
\hline MOL001506 & Supraene & 33.55 & 0.42 & 2.08 \\
\hline MOL003759 & Iristectorigenin A & 63.36 & 0.34 & 0.54 \\
\hline MOL003975 & Icosa-11,14,17-trienoic acid methyl ester & 44.81 & 0.23 & 1.52 \\
\hline MOL006630 & Norartocarpetin & 54.93 & 0.24 & 0.14 \\
\hline MOL007179 & Linolenic acid ethyl ester & 46.1 & 0.2 & 1.48 \\
\hline MOL007879 & Tetramethoxyluteolin & 43.68 & 0.37 & 0.96 \\
\hline
\end{tabular}

180 disease targets were obtained for subsequent analysis (Fig. 2A). The herb targets were intersected with the disease targets to obtain 24 MLE-obesity-related targets by Venny (version 2.1; https://bioinfogp.cnb.csic.es/tools/venny/index. html; Fig. 2B). As shown in Fig. 2C, a network of 24 interacting proteins consisted of 34 edges. The average node degree was 2.83 and the PPI enrichment P-value was $5.49 \times 10^{-12}$. TNF, PPARG, prostaglandin-endoperoxide synthase 2 (PTGS2), nuclear receptor subfamily 3 group $\mathrm{C}$ member 1 and histone deacetylase 3 (HDAC3) had high degree of the nodes. In addition, an MLE-compounds-targets-obesity interaction network was constructed to facilitate the understanding of the potential regulatory effects of MLE holistically (Fig. 2D). In total, four compounds had no corresponding targets found in the database, therefore only the interaction network of 22 compounds and their relevant targets was shown. In this network, arachidonic acid had 10 targets, whereas isoramanone, icosa-11,14,17-trienoic acid methyl ester and linolenic acid ethyl ester all had nine targets each. These multi-target compounds may serve to be the key active components of MLE. Fatty acid oxidation associated proteins (HSD11B1), kinase insert domain receptor (KDR), phosphodiesterase 4D (PDE4D), PPARD, PPARG, PTGS2 and FAAH had high degree of the nodes in the network, which may be the core candidate targets of MLE.
PPI, GO and KEGG analysis. There were 234 adjacent genes of 24 MLE-obesity related targets identified by STRING. A PPI network analysis of the 234 adjacent genes of the 24 targets was conducted using STRING to explore the potential interactions among them (Fig. 3A). GO functional enrichment analysis was subsequently performed and the target enrichment was relatively concentrated in the biological processes of 'regulation of lipid metabolic process', 'response to nutrient levels', 'fatty acid metabolic process', 'response to steroid hormone', 'regulation of inflammatory response', 'lipid catabolic process' and 'fatty acid biosynthetic process' (Fig. 3B). Additionally, KEGG pathways of the 234 adjacent genes were analyzed and shown in Fig. 3C. The pathways included 'adipocytokine signaling pathway', 'TNF signaling pathway', 'Regulation of lipolysis in adipocytes', 'NF-kB signaling pathway', 'insulin resistance', 'PPAR signaling pathway' and 'AMPK signaling pathway'. In general, these biological processes and signaling pathways were likely to be associated with the beneficial effects of MLE against obesity.

MLE treatment of high-fat diet-induced obese mice. The weight of mice was found to be significantly increased in the Model group compared with that in the control group but significantly reversed by both low- and high-dose MLE treatment at 8 weeks (Fig. 4A). The serum levels of TC and TG 
A
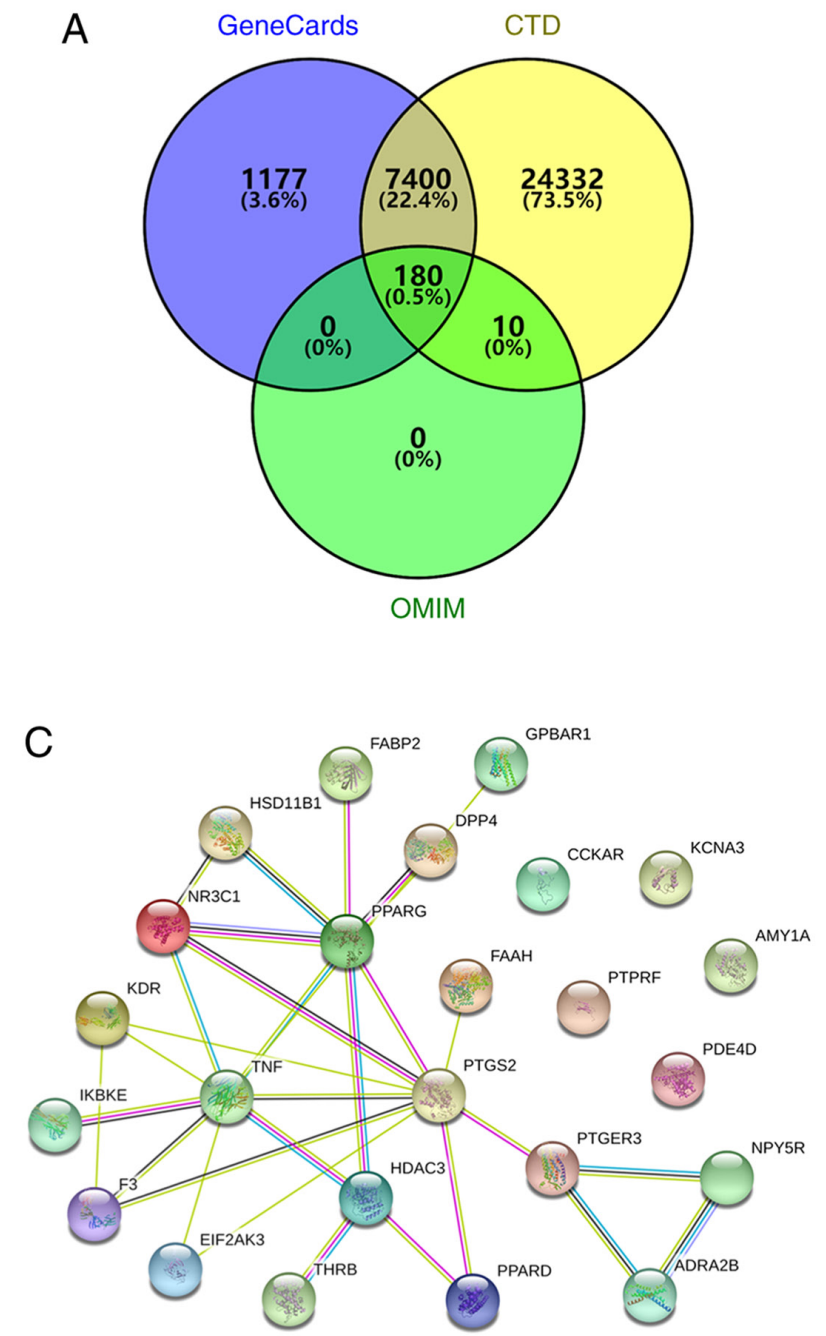

B
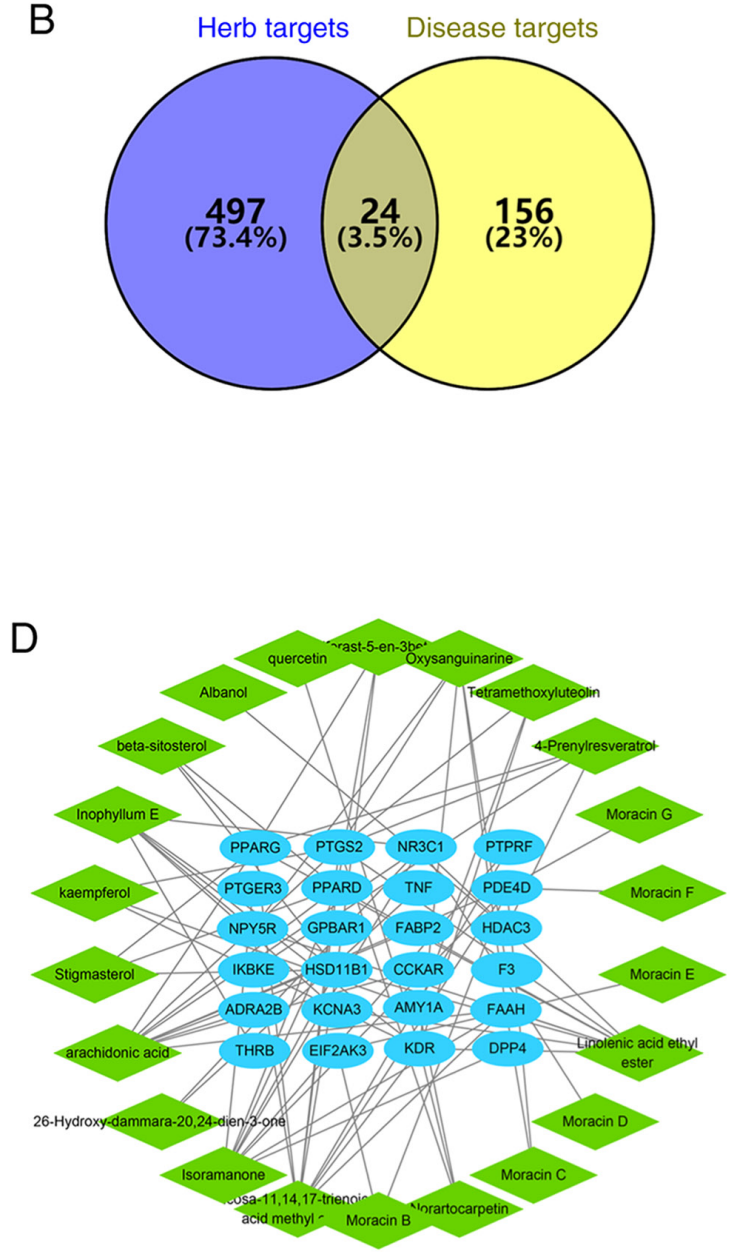

Figure 2. Active components and potential target proteins for MLE. (A) In total, 180 obesity-related genes were intersected among OMIM, GeneCards and CTD databases by Venny. (B) The intersection of the potential targets of MLE on obesity were taken using Venny. (C) Protein-protein interaction network of the potential targets of MLE on obesity was presented by Search Tool for the Retrieval of Interacting Genes/Proteins. (D) MLE compounds-targets-obesity interaction network was performed through Cytoscape. The green diamond represented the candidate compound, and the blue ellipse was the target. The number of edges connecting to the nodes determined the degree value of the nodes. MLE, Mulberry leaf extract; CTD, Comparative Toxicogenomics Database; OMIM, Online Mendelian Inheritance in Man.

of the Model group were also significantly upregulated, which were also significantly reversed by both doses MLE treatment, although the effect of high-dose MLE on the TC levels was more prominent (Fig. 4B). Hepatic histological examination demonstrated that fat deposition (the white area represents fat) was increased in the Model group compared with that in the Control group. MLE treatment, especially at higher doses, was associated with markedly reduced fat accumulation compared with that in the Model group (Fig. 4C). According to Oil red O staining, higher numbers of lipid droplets could be observed in the liver of high-fat diet-induced obese mice compared with those in the Control group, whilst addition of MLE markedly decreased the hepatic lipid droplets at both doses (Fig. 4D).

Effects of MLE on inflammation, lipogenesis, lipid catabolism, fatty acid oxidation and the AMP-activated protein kinase (AMPK) signaling pathway. The inflammatory factors (TNF- $\alpha$, IL-1 $\beta$, NFKBIA and iNOS), fat synthesis related proteins (PPARD and PPARG), lipid catabolism related protein $(\mathrm{FAAH})$ and fatty acid oxidation associated proteins
(HSD11B1) were chosen from KEGG analysis. The protein expression levels of TNF- $\alpha$, IL-1 $\beta$, NFKBIA and iNOS in visceral white adipose tissues were all significantly increased in the Model group compared with those in the Control group, which were all significantly reversed by treatment with of MLE in a dose-dependent manner (Fig. 5A). The mRNA expression levels of TNF- $\alpha$, PPARD and PPARG were all significantly increased, whereas those of FAAH and HSD11B1 were significantly decreased in the visceral white adipose tissues of mice in the Model group compared with those in the Control group (Fig. 5B). However, these effects were also markedly reversed by MLE, with significant reversals observed on all mRNAs analyzed following high-dose MLE treatment (Fig. 5B). The levels of phosphorylated (p-)AMPK/AMPK were significantly reduced, whilst the expression levels of PPARG, SREBP-1 and FAS were significantly increased in the model group compared with those in the Control group. Treatment with the high-dose MLE significantly reversed the aforementioned changes in AMPK phosphorylation and PPARG, SREBP-1 and FAS expression (Fig. 5C). 
A

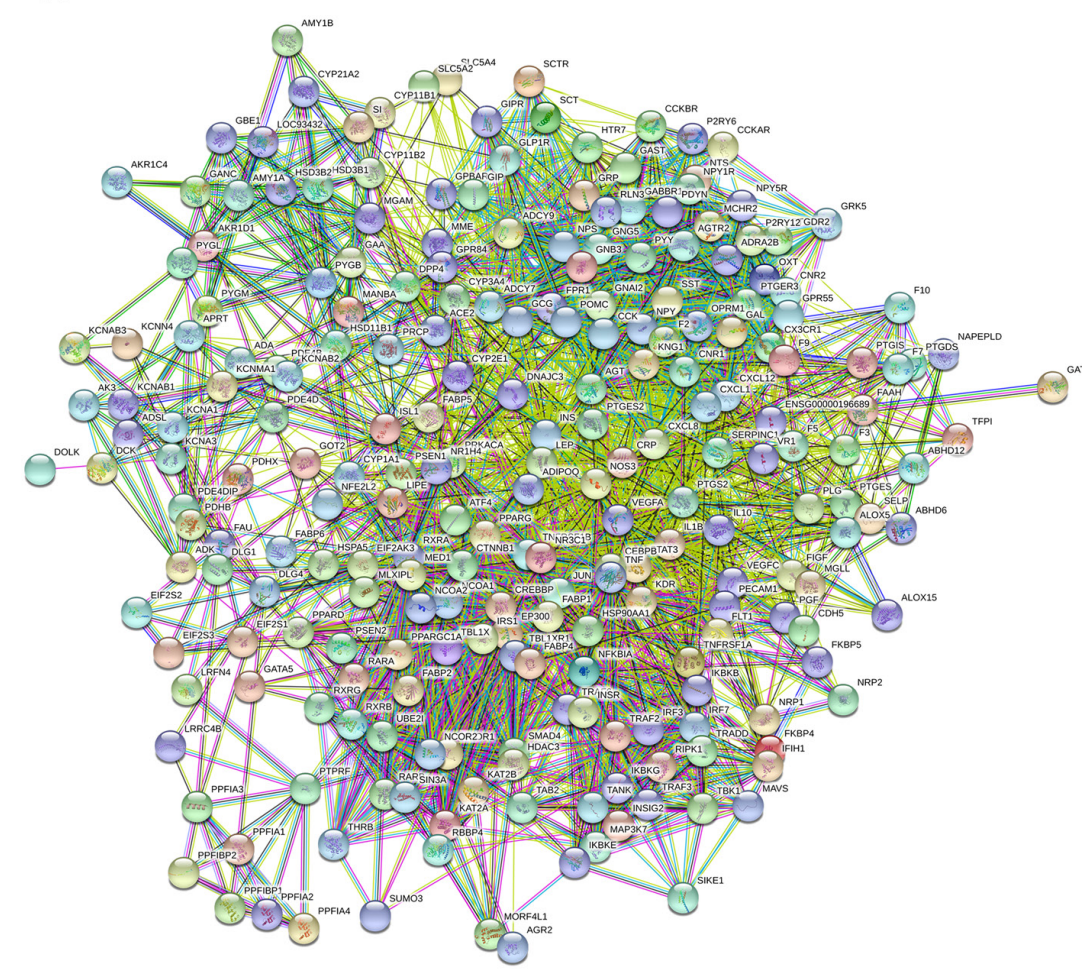

B

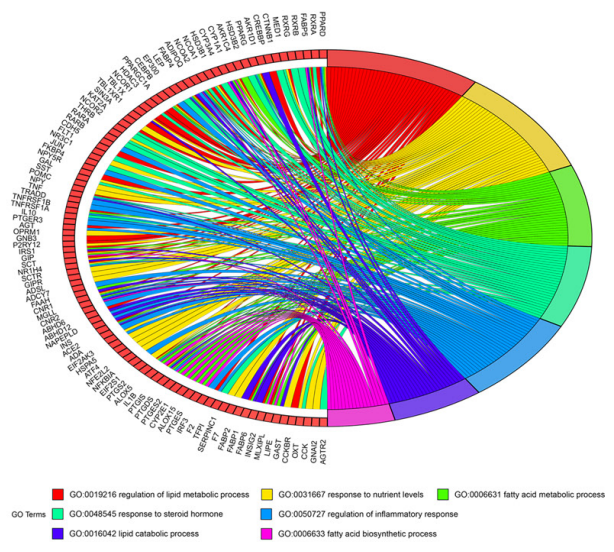

C

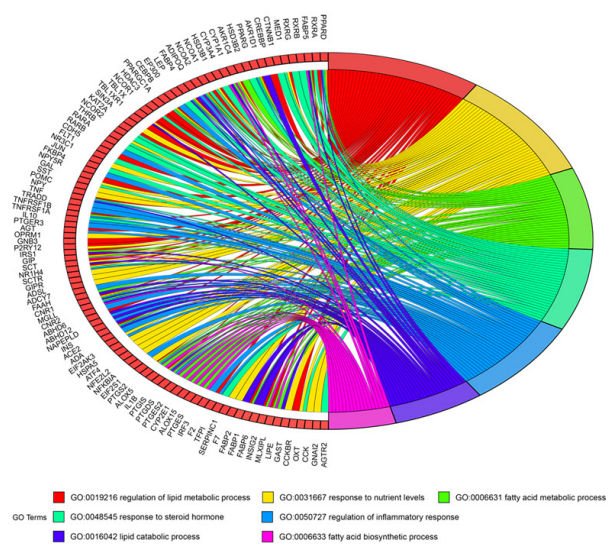

Figure 3. PPI, GO and KEGG analysis of target proteins. (A) PPI network analysis of the 234 adjacent genes of the 24 targets using STRING. (B) GO enrichment and (C) KEGG pathway analysis of the target proteins by the 'clusterProfiler' package in R. PPI, Protein-protein interaction; GO, gene ontology; KEGG, Kyoto Encyclopedia of Genes and Genomes; STRING, Search Tool for the Retrieval of Interacting Genes/Proteins.

\section{Discussion}

The effects of specific active components contained within certain traditional Chinese medicines on mammalian cells and gene expression remain unclear. Therefore, it is necessary to form a knowledge base associated with these compounds (21). The emergence of big data in the biomedical research field has promoted the establishment of network pharmacology for the systematic analysis of drug targets. Additionally, it changed the concept of drug discovery from 'a single target, a single drug' to 'multi-targets, multi-components' (22). Network pharmacology is an emerging discipline that is based on the statistical analysis of data, virtual computing and the observation of component-target-disease-pathway interactions retrieved from network databases (23).

MLE have been previously demonstrated to alleviate hyperglycemia, hyperlipidemia, obesity, oxidation and inflammation (24-28). Pharmacological analysis has indicated that the active components in MLE include $\beta$-carotene, quercetin, $\beta$-sitosterol, kaempferol and arachidonic acid, all of which can exert beneficial effects against obesity (29-33). Using network pharmacological analysis, we speculated that these components can alleviate high-fat diet-induced obesity by targeting proteins in various metabolic pathways. Furthermore, the majority of the identified pathways are predominantly involved in glucolipid metabolism, inflammation, lipogenesis and fatty acid oxidation, which should be explored in future studies.
A previous study has revealed that obesity is associated with chronic low-grade inflammation, such that insulin signaling may be interfered by inflammatory factors, leading to insulin resistance and metabolic disorders, including type 2 diabetes and metabolic syndrome, where impairments in insulin signaling disrupt the entry of glucose into the adipocytes and skeletal muscle cells (34). Adipocytes in the adipose tissues of obese individuals are frequently found to be enlarged, which is accompanied with increased tissue vascularization, which affects the utilization of oxygen and leads to oxygen deficiency in the adipose tissue (35). In particular, hypoxia-inducible factor- $1 \alpha$ is associated with chronic inflammation of the adipose tissue by promoting the recruitment of macrophages and their transformation to the proinflammatory M1 type, which releases a variety of proinflammatory cytokines, including TNF- $\alpha$ and IL- 6 in obesity (36). During obesity, inflammation occurs not only in the adipose tissue, but also in other tissues (37). It has been previously reported that obese mice fed with a high-fat diet exhibit intestinal inflammation, elevated levels of TNF- $\alpha$ and activation of the NF- $\kappa \mathrm{B}$ signaling pathway (38). In the present study, it was observed that the protein expression levels of inflammatory factors TNF- $\alpha$, IL- $1 \beta$, NFKBIA and iNOS were also increased in the adipose tissues of obese mice. Additionally, 'TNF signaling pathway', 'NF- $\mathrm{B}$ signaling pathway' and 'insulin resistance' were identified by KEGG pathway enrichment analysis, suggesting that MLE may exert 

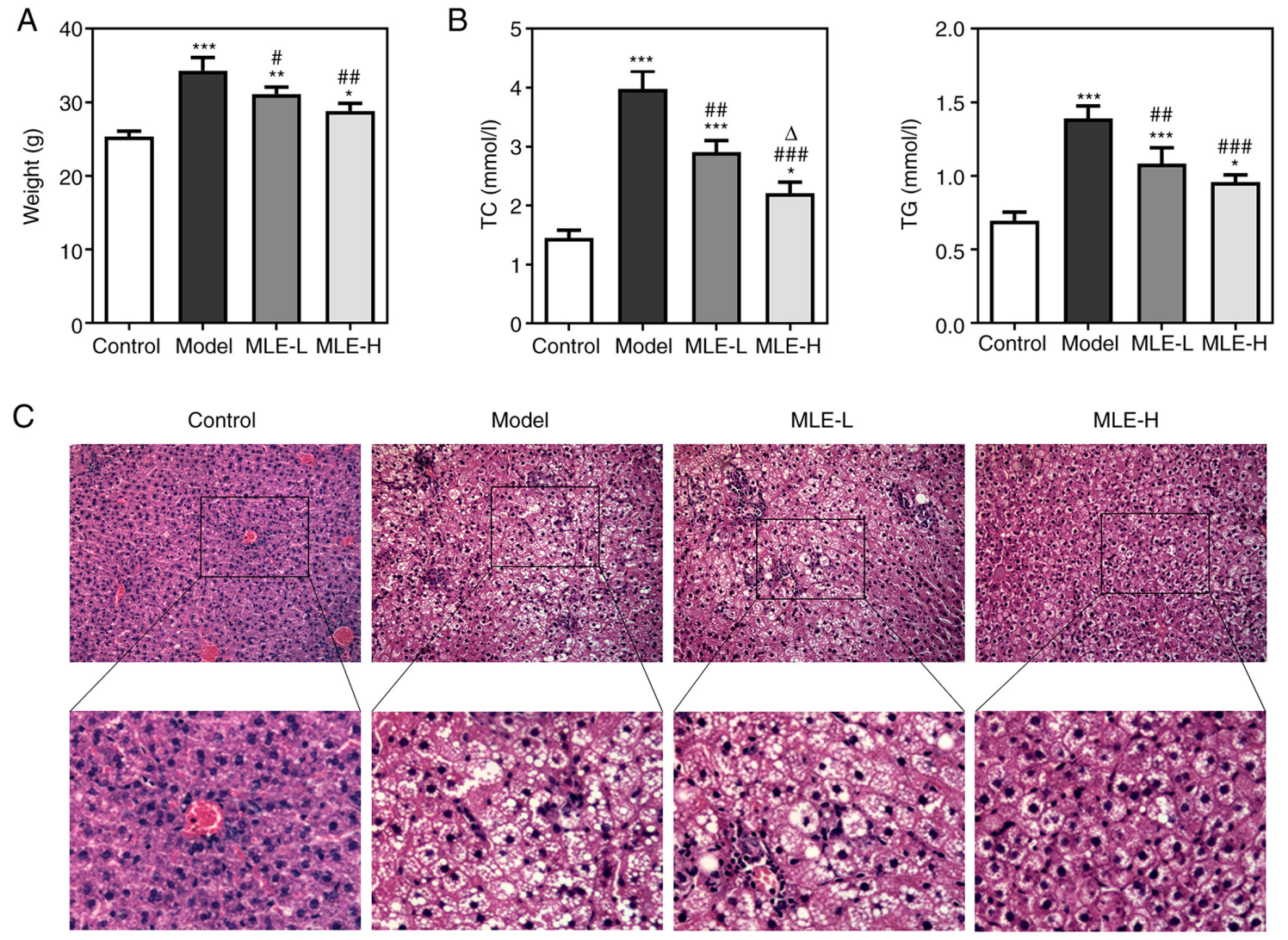

D

Control

Model

MLE-L

MLE-H
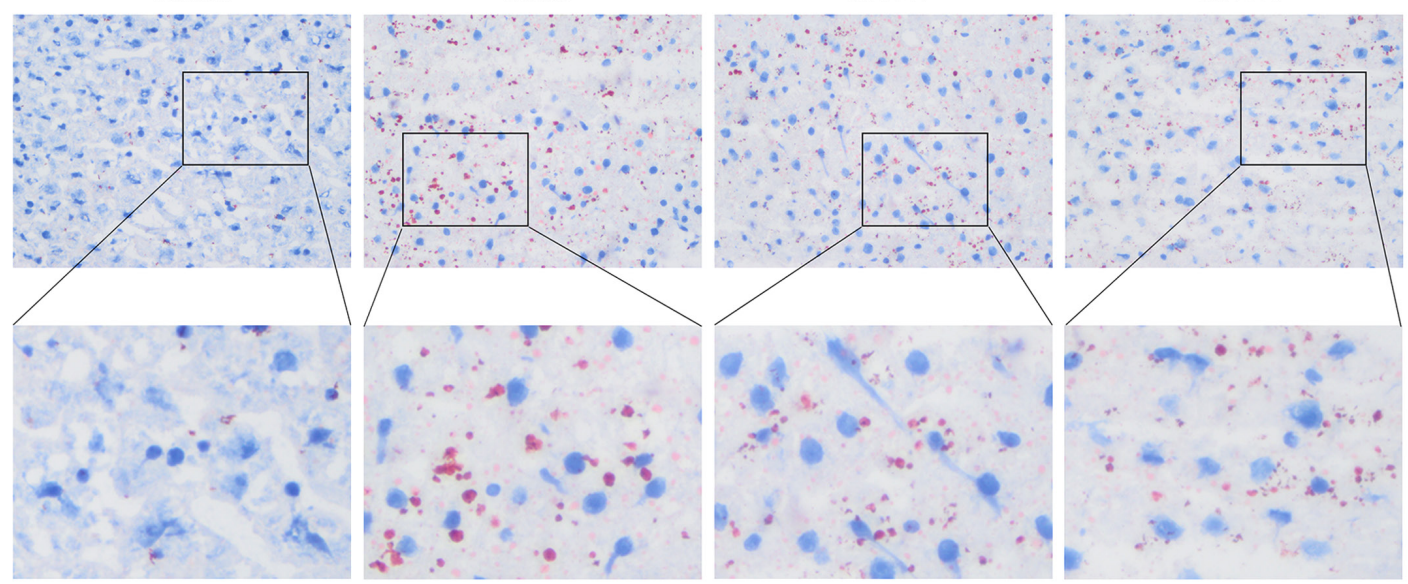

Figure 4. Effects of MLE on high-fat diet-induced obese mice. (A) Mice body weight at 8 weeks. (B) The levels of serum TC and TG were measured using TC and TG assay kits. (C) Hepatic fat accumulation was detected by H\&E staining. Original (magnification, $\mathrm{x} 200$ ) and blow-up (magnification, $\mathrm{x} 400$ ). (D) Liver lipid droplets were detected by Oil red $\mathrm{O}$ staining. Original (magnification, $\mathrm{x} 400$ ) and blow-up (magnification, $\mathrm{x} 800$ ). ${ }^{*} \mathrm{P}<0.05,{ }^{* * *} \mathrm{P}<0.01$ and ${ }^{* * * *} \mathrm{P}<0.001$ vs. Control; ${ }^{\#} \mathrm{P}<0.05,{ }^{\# \#} \mathrm{P}<0.01$ and ${ }^{\# \# \#} \mathrm{P}<0.001$ vs. Model; ${ }^{\Delta} \mathrm{P}<0.05$ vs. MLE-L. TC, total cholesterol; TG, triglyceride; MLE, Mulberry leaf extract; MLE-L, low dose MLE; MLE-H, high dose MLE.

beneficial effects in the treatment of obesity through the signaling pathways aforementioned.

The first indication for increased cytokine release in obesity is increased TNF- $\alpha$ expression, the levels of which are associated with the degree of adiposity and insulin resistance (39). The rs2016520 polymorphism of PPARD has been revealed to serve a leading role in the development of abdominal obesity (40). In addition, obesity-susceptibility gene transmembrane protein 18 can upregulate PPARG expression to promote adipogenesis during obesity (41). FAAH is a primary catabolic regulator of N-acylethanolamines, which activates $\mathrm{G}$-protein-coupled receptors within the endocannabinoid system (42). FAAH is associated with increased BMI, increased triglyceride levels and reduced levels of high-density lipoprotein cholesterol, which occurs during obesity (42). Hydroxysteroid (11- $\beta$ ) dehydrogenase type 1 (11- $\beta$ HSD1) is a bidirectional enzyme encoded by the HSD11B1 gene and is highly expressed in the liver and adipose tissues (43). It can convert inactive cortisone into its active form cortisol (43). Individuals with obesity typically show decreased HSD11B1 gene expression in the intra-abdominal visceral adipose tissue (VAT) (44). Therefore, these obesity-related genes (TNF- $\alpha$, PPARD, PPARG, FAAH and HSD11B1) were selected to verify the effects of treatment with MLE on obesity. The results indicated that the expression 
A
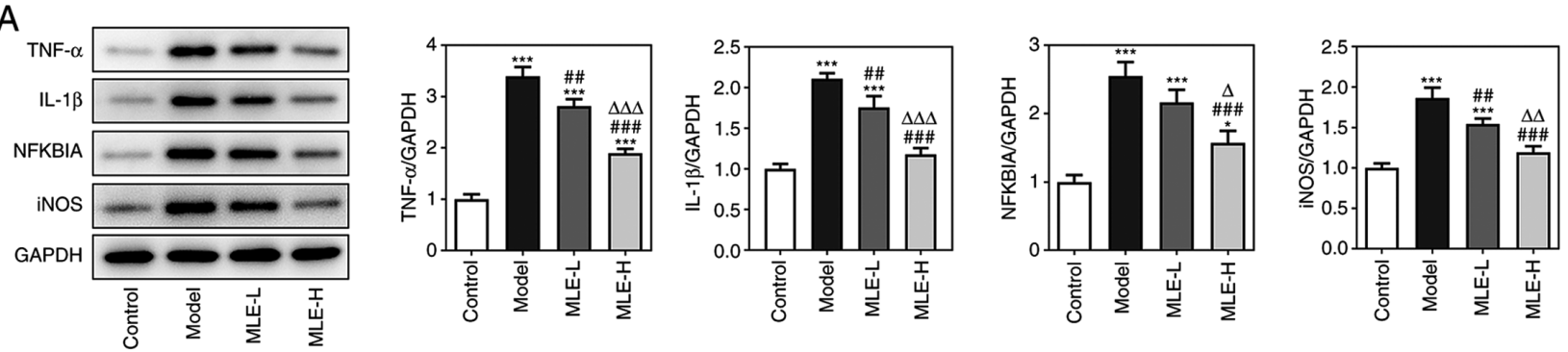

B
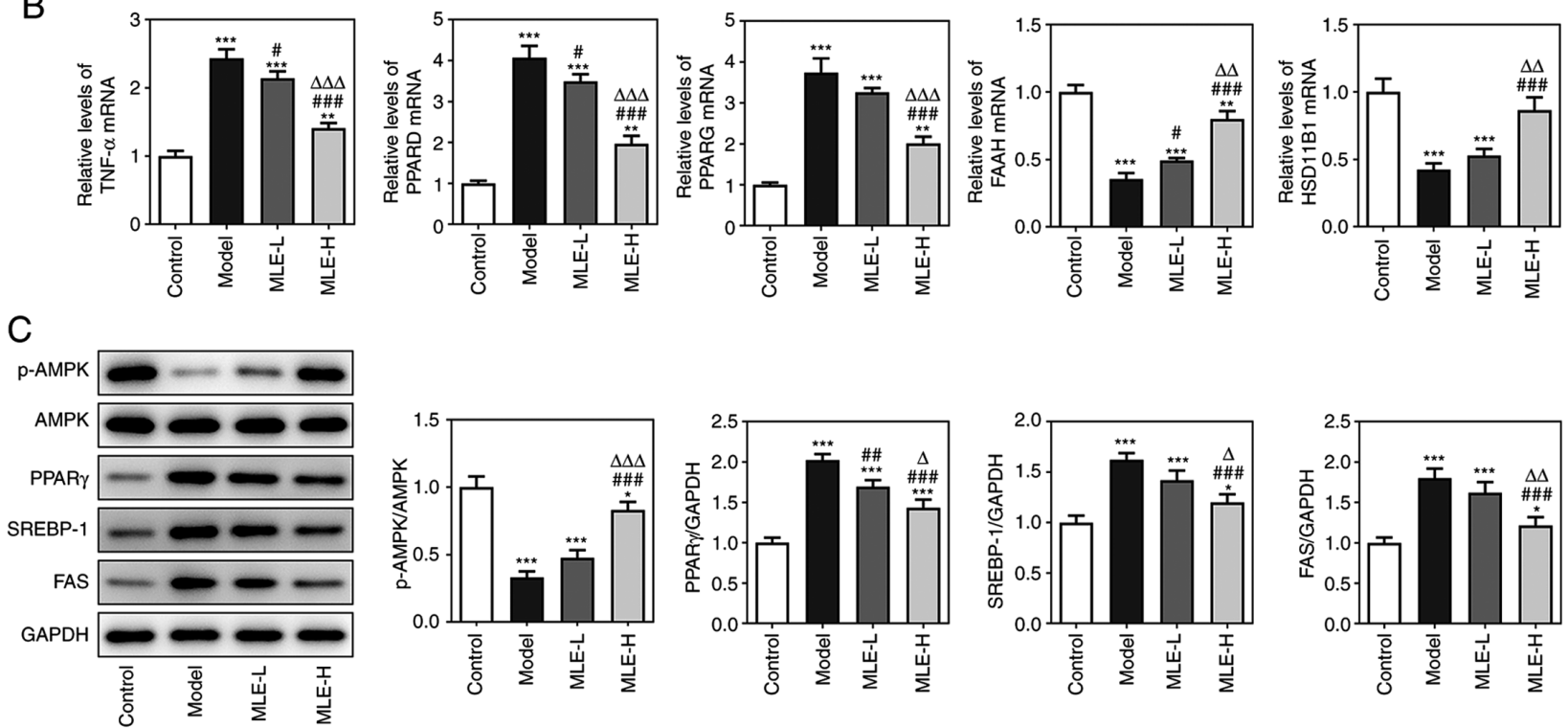

Figure 5. Effects of MLE on adipocyte inflammation, lipogenesis, lipid catabolism, fatty acid oxidation and AMPK signaling. (A) The protein expressions of inflammatory factors TNF- $\alpha$, IL-1 $\beta$, NFKBIA and iNOS in adipose tissue were detected by western blot analysis. (B) The mRNA expression of key targets of MLE in the treatment of obesity was verified by reverse transcription-quantitative PCR. (C) The activity of the AMPK pathway in adipose tissue was detected by

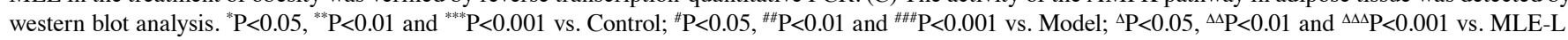
MLE, Mulberry leaf extract; MLE-L, low dose MLE; MLE-H, high dose MLE; NFKBIA, NF-кB inhibitor $\alpha$; iNOS, inducible nitric oxide synthase; AMPK, 5'AMP-activated protein kinase; PPAR, peroxisome proliferator activated receptor; SREBP-1, Sterol regulatory element binding protein-1; FAS, fatty acid synthase.

levels of TNF- $\alpha$, PPARD and PPARG were increased, whilst the expression levels of FAAH and HSD11B1 were decreased by obesity, which were markedly reversed by MLE treatment. The altered expression levels of TNF- $\alpha$, PPARD, PPARG, FAAH and HSD11B1 in obesity treated with MLE were consistent with results from previous studies aforementioned.

AMPK is a cellular energy sensor that functions as a serine/threonine specific protein kinase (45). It serves a key role in maintaining the metabolic balance in the body $(46,47)$. Activation of AMPK can increase catabolism, reduce the expression levels of regulators of lipid synthesis, including acetyl-CoA carboxylase (ACC), FAS and SREBP, to reduce the rate of anabolism whilst regulating lipid synthesis and utilization (48). PPAR $\gamma$ is a major transcription factor that can modulate glucose and lipid metabolism, the expression of which in adipose tissues has been previously associated with high fat diet-induced adipocyte hypertrophy and insulin resistance $(49,50)$. SREBP-1c is a transcription factor that regulates lipogenesis (51). AMPK-knockout mice frequently develop metabolic complications, such as dysregulated glucose homeostasis and insulin resistance, when they are fed a high-fat diet (52). However, activation of AMPK using A-769662 has been found to alleviate metabolic dysfunction in mice fed a high-fat diet (52). AMPK can reduce de novo lipogenesis and promote fatty acid oxidation and inhibition of ACC phosphorylation and SREBP-1c activation by AMPK can inhibit fatty-acid synthesis (53). In the present study, it was observed that p-AMPK/AMPK levels were decreased, whilst the expression levels of PPAR $\gamma$, SREBP-1 and FAS were increased by obesity, which were markedly reversed by MLE treatment.

However, the present study has some limitations. Although the present study suggested that MLE negatively regulated inflammation, lipogenesis, lipid catabolism, fatty acid oxidation and AMPK signaling, whether this effect was indeed mediated by these signaling pathways should be improved using related inhibitors, gene knockout or overexpression.

In conclusion, a network pharmacology approach was used in the present study to identify active components of MLE and their potential target proteins associated with obesity. The results indicated that 24 targets associated with obesity were screened to be targets for MLE, where seven related biological processes and seven related signaling pathways were extracted by GO and KEGG analyzes, respectively. Subsequently, an in vivo experiment was used to verify the network pharmacology analysis and revealed that the MLE-induced obesity improvement may be due to the upregulation of FAAH and HSD11B1 and downregulation of TNF, PPAR $\delta$ and PPAR $\gamma$. 


\section{Acknowledgements}

Not applicable.

\section{Funding}

No funding was received.

\section{Availability of data and materials}

The datasets used and/or analyzed during the current study are available from the corresponding author on reasonable request.

\section{Authors' contributions}

JD conceived and designed the study. GW performed the experiments, analyzed the data and drafted the manuscript. JD and GW confirm the authenticity of all raw data. All authors read and approved the final manuscript.

\section{Ethics approval and consent to participate}

The experimental protocols of the present study were approved by the Institutional Animal Care and Use Committee of the Hunan Future Health Technology Group Co., Ltd. (Changsha, China).

\section{Patient consent for publication}

Not applicable.

\section{Competing interests}

The authors declare they have no competing interests.

\section{References}

1. Tsai AG and Bessesen DH: Obesity. Ann Intern Med 170 ITC33-ITC48, 2019.

2. Jia A, Xu S, Ming J, et al: Epidemic characteristics of obesity in China under various diagnostic criteria. Chin J Diabetes Mellitus 9: 221-225, 2017 (In Chinese).

3. Kim YJ, Lee HS, Kim YK, Park S, Kim JM, Yun JH, Yu HY and Kim BJ: Association of metabolites with obesity and Type 2 diabetes based on FTO genotype. PLoS One 11: e0156612, 2016

4. Lavie CJ, De Schutter A, Parto P, Jahangir E, Kokkinos P, Ortega FB, Arena R and Milani RV: Obesity and prevalence of cardiovascular diseases and Prognosis-The obesity paradox updated. Prog Cardiovasc Dis 58: 537-547, 2016.

5. Yang XD, Jiang S, Wang G, Zhang R, Zhang J and Zhu JS: Link of obesity and gastrointestinal cancer: Crossroad of inflammation and oxidative stress. J Biol Regul Homeost Agents 29: 755-760, 2015.

6. Wang R, Li Y, Mu W, Li Z, Sun J, Wang B, Zhong Z, Luo X, $\mathrm{Xie} \mathrm{C}$ and Huang Y: Mulberry leaf extract reduces the glycemic indexes of four common dietary carbohydrates. Medicine (Baltimore 97: e11996, 2018.

7. Park JM, Bong HY, Jeong HI, Kim YK, Kim JY and Kwon O: Postprandial hypoglycemic effect of mulberry leaf in Goto-Kakizaki rats and counterpart control Wistar rats. Nutr Res Pract 3: 272-278, 2009.

8. Yang X, Yang L and Zheng H: Hypolipidemic and antioxidant effects of mulberry (Morus alba L.) fruit in hyperlipidaemia rats. Food Chem Toxicol 48: 2374-2379, 2010.
9. Andallu B, Suryakantham V, Lakshmi Srikanthi B and Reddy GK: Effect of mulberry (Morus indica L.) therapy on plasma and erythrocyte membrane lipids in patients with type 2 diabetes. Clin Chim Acta 314: 47-53, 2001.

10. Arabshahi-Delouee S and Urooj A: Antioxidant properties of various solvent extracts of mulberry (Morus indica L.) leaves. Food Chem 102: 1233-1240, 2007.

11. Ramadan EM, Abou-Taleb KA, Galal GF and Abdel-Hamid SN: Antibacterial, antibiofilm and antitumor activities of grape and mulberry leaves ethanolic extracts towards bacterial clinical strains. Ann Agric Sci 62: 151-159, 2017.

12. Zeni AL and Dall'Molin M: Hypotriglyceridemic effect of Morus alba L., Moraceae, leaves in hyperlipidemic rats. Rev Bras Farmacogn 20: 130-133, 2010.

13. Lee YJ, Choi DH, Kim EJ, Kim HY, Kwon TO, Kang DG and Lee HS: Hypotensive, hypolipidemic, and vascular protective effects of Morus alba L. in rats fed an atherogenic diet. Am J Chin Med 39: 39-52, 2011.

14. Peng CH, Lin HT, Chung DJ, Huang CN and Wang CJ: Mulberry leaf extracts prevent obesity-induced NAFLD with regulating adipocytokines, inflammation and oxidative stress. J Food Drug Anal 26: 778-787, 2018.

15. Sheng Y, Liu J, Zheng S, Liang F, Luo Y, Huang K, Xu W and He X: Mulberry leaves ameliorate obesity through enhancing brown adipose tissue activity and modulating gut microbiota. Food Funct 10: 4771-4781, 2019.

16. Li Q, Liu F, Liu J, Liao S and Zou Y: Mulberry leaf polyphenols and fiber induce synergistic antiobesity and display a modulation effect on gut microbiota and metabolites. Nutrients 11: 1017, 2019.

17. Yu G, Wang LG, Han Y and He QY: ClusterProfiler: An Rpackage for comparing biological themes among gene clusters. OMICS 16: 284-287, 2012.

18. Walter W, Sánchez-Cabo F and Ricote M: GOplot: An R package for visually combining expression data with functional analysis. Bioinformatics 31: 2912-2914, 2015.

19. Ann JY, Eo H and Lim Y: Mulberry leaves (Morus alba L.) ameliorate obesity-induced hepatic lipogenesis, fibrosis, and oxidative stress in high-fat diet-fed mice. Genes Nutr 10: 46, 2015.

20. Livak KJ and Schmittgen TD: Analysis of relative gene expression data using real-time quantitative PCR and the 2(-Delta Delta C(T)) Method. Methods 25: 402-408, 2001.

21. Zhang RZ, Yu SJ, Bai H and Ning K: TCM-Mesh: The database and analytical system for network pharmacology analysis for TCM preparations. Sci Rep 7: 2821, 2017.

22. Li S: Network target: A starting point for traditional Chinese medicine network pharmacology. Zhongguo Zhong Yao Za Zhi 36: 2017-2020, 2011 (In Chinese).

23. Tang H, He S, Zhang X, Luo S, Zhang B, Duan X, Zhang Z, Wang W, Wang Y and Sun Y: A network pharmacology approach to uncover the pharmacological mechanism of XuanHuSuo powder on osteoarthritis. Evid Based Complement Alternat Med 2016: 3246946, 2016.

24. Kim GN, Kwon YI and Jang HD: Mulberry leaf extract reduces postprandial hyperglycemia with few side effects by inhibiting $\alpha$-glucosidase in normal rats. J Med Food 14: 712-717, 2011.

25. Lee HJ, Na YG, Han M, Pham TMA, Lee H, Lee HK, Myung CS, Han JH, Kang JS, Kim KT and Cho CW: Statistical design of sustained-release tablet garcinia cambogia extract and bioconverted mulberry leaf extract for anti-obesity. Pharmaceutics 12: 932, 2020.

26. Aramwit P, Supasyndh O, Siritienthong T and Bang N: Mulberry leaf reduces oxidation and C-reactive protein level in patients with mild dyslipidemia. Biomed Res Int 2013: 787981, 2013.

27. Huang J, Wang Y, Ying C, Liu L and Lou Z: Effects of mulberry leaf on experimental hyperlipidemia rats induced by high-fat diet. Exp Ther Med 16: 547-556, 2018.

28. Tian S, Wang M, Liu C, Zhao H and Zhao B: Mulberry leaf reduces inflammation and insulin resistance in type 2 diabetic mice by TLRs and insulin signalling pathway. BMC Complement Altern Med 19: 326, 2019.

29. Marcelino G, Machate DJ, Freitas KC, Hiane PA, Maldonade IR, Pott A, Asato MA, Candido CJ and Guimarães RCA: $\beta$-Carotene: Preventive role for type 2 diabetes mellitus and obesity: A review. Molecules 25: 5803, 2020.

30. Jiang H, Horiuchi Y, Hironao KY, Kitakaze T, Yamashita Y and Ashida H: Prevention effect of quercetin and its glycosides on obesity and hyperglycemia through activating AMPK $\alpha$ in high-fat diet-fed ICR mice. J Clin Biochem Nutr 67: 74-83, 2020. 
31. Kurano M, Hasegawa K, Kunimi M, Hara M, Yatomi Y, Teramoto T and Tsukamoto K: Sitosterol prevents obesity-related chronic inflammation. Biochim Biophys Acta Mol Cell Biol Lipids 1863: 191-198, 2018.

32. Wang T, Wu Q and Zhao T: Preventive effects of kaempferol on high-fat diet-induced obesity complications in C57BL/6 mice. Biomed Res Int 2020: 4532482, 2020

33. Mak IL, Lavery P, Agellon S, Rauch F, Murshed M and Weiler HA: Arachidonic acid exacerbates diet-induced obesity and reduces bone mineral content without impacting bone strength in growing male rats. J Nutr Biochem 73: 108226, 2019.

34. Yaribeygi H, Farrokhi FR, Butler AE and Sahebkar A: Insulin resistance: Review of the underlying molecular mechanisms. J Cell Physiol 234: 8152-8161, 2019.

35. Hammarstedt A, Gogg S, Hedjazifar S, Nerstedt A and Smith U: Impaired adipogenesis and dysfunctional adipose tissue in human hypertrophic obesity. Physiol Rev 98: 1911-1941, 2018.

36. Poblete JMS, Ballinger MN, Bao S, Alghothani M, Nevado JB Jr, Eubank TD, Christman JW and Magalang UJ: Macrophage HIF-1 $\alpha$ mediates obesity-related adipose tissue dysfunction via interleukin-1 receptor-associated kinase M. Am J Physiol Endocrinol Metab 318: E689-E700, 2020.

37. Yao L, Bhatta A, Xu Z, Chen J, Toque HA, Chen Y, Xu Y, Bagi Z, Lucas R, Huo Y, et al: Obesity-induced vascular inflammation involves elevated arginase activity. Am J Physiol Regul Integr Comp Physiol 313: R560-R571, 2017.

38. Ding S, Chi MM, Scull BP, Rigby R, Schwerbrock NM, Magness S, Jobin C and Lund PK: High-fat diet: Bacteria interactions promote intestinal inflammation which precedes and correlates with obesity and insulin resistance in mouse. PLoS One 5: e12191, 2010

39. Tzanavari T, Giannogonas P and Karalis KP: TNF-alpha and obesity. Curr Dir Autoimmun 11: 145-156, 2010.

40. Ding Y, Guo ZR, Wu M, Chen Q, Zhou ZY, Yu H, Zhang LJ, Liu JC and Luo WS: Effects of PPARD-87T $>C$ and interactions with single nucleotide polymorphisms in PPARA and PPARG on abdominal obesity. Zhonghua Yi Xue Za Zhi 92: 1517-1521, 2012 (In Chinese).

41. Landgraf K, Klöting N, Gericke M, Maixner N, Guiu-Jurado E, Scholz M, Witte AV, Beyer F, Schwartze JT, Lacher M, et al: The obesity-susceptibility gene TMEM18 promotes adipogenesis through activation of PPARG. Cell Rep 33: 108295, 2020.

42. Zhang Y, Sonnenberg GE, Baye TM, Littrell J, Gunnell J, DeLaForest A, MacKinney E, Hillard CJ,Kissebah AH,Olivier M and Wilke RA: Obesity-related dyslipidemia associated with FAAH, independent of insulin response, in multigenerational families of Northern European descent. Pharmacogenomics 10: 1929-1939, 2009.
43. Tomlinson JW, Moore JS, Clark PM, Holder G, Shakespeare L and Stewart PM: Weight loss increases 11beta-hydroxysteroid dehydrogenase type 1 expression in human adipose tissue. J Clin Endocrinol Metab 89: 2711-2716, 2004.

44. Chedid MF, do Nascimento FV, de Oliveira FS, de Souza BM, Kruel CR, Gurski RR, Canani LH, Crispim D and Gerchman F: Interaction of HSD11B1 and H6PD polymorphisms in subjects with type 2 diabetes are protective factors against obesity: A cross-sectional study. Diabetol Metab Syndr 11: 78, 2019.

45. Zhu XJ, Dai JQ, Tan X, Zhao Y and Yang WJ: Activation of an AMP-activated protein kinase is involved in post-diapause development of Artemia franciscana encysted embryos. BMC Dev Biol 9: 21, 2009.

46. Hardie DG: New roles for the LKB1->AMPK pathway. Curr Opin Cell Biol 17: 167-173, 2005.

47. Hardie DG: The AMP-activated protein kinase pathway-new players upstream and downstream. J Cell Sci 117: 5479-5487, 2004.

48. Herzig S and Shaw RJ: AMPK: Guardian of metabolism and mitochondrial homeostasis. Nat Rev Mol Cell Biol 19: 121-135, 2018.

49. Kubota N, Terauchi Y, Miki H, Tamemoto H, Yamauchi T, Komeda K, Satoh S, Nakano R, Ishii C, Sugiyama T, et al: PPAR gamma mediates high-fat diet-induced adipocyte hypertrophy and insulin resistance. Mol Cell 4: 597-609, 1999.

50. Jones JR, Barrick C, Kim KA, Lindner J, Blondeau B, Fujimoto Y, Shiota M, Kesterson RA, Kahn BB and Magnuson MA: Deletion of PPARgamma in adipose tissues of mice protects against high fat diet-induced obesity and insulin resistance. Proc Natl Acad Sci USA 102: 6207-6212, 2005.

51. Crewe C, Zhu Y, Paschoal VA, Joffin N, Ghaben AL, Gordillo R, Oh DY, Liang G, Horton JD and Scherer PE: SREBP-regulated adipocyte lipogenesis is dependent on substrate availability and redox modulation of mTORC1. JCI insight 5: e129397, 2019.

52. Wu L, Zhang L, Li B, Jiang H, Duan Y, Xie Z, Shuai L, Li J and Li J: AMP-Activated Protein Kinase (AMPK) regulates energy metabolism through modulating thermogenesis in adipose tissue. Front Physiol 9: 122, 2018.

53. Jeon SM: Regulation and function of AMPK in physiology and diseases. Exp Mol Med 48: e245, 2016.

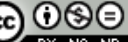

This work is licensed under a Creative Commons Attribution-NonCommercial-NoDerivatives 4.0 International (CC BY-NC-ND 4.0) License. 